The final publication is available at Elsevier via https://doi.org/10.1016/j.engstruct.2019.05.064. @ 2019. This manuscript version is made available under the CC-BY-NC-ND 4.0 license http://creativecommons.org/licenses/by-nc-nd/4.0/

\title{
Storey-Based Stability of Unbraced Steel Frames under Piece- Linear Temperature Distributions
}

\author{
Terence $\mathrm{Ma}^{1}$, Lei $\mathrm{Xu}^{1}$ \\ ${ }^{1}$ Department of Civil and Environmental Engineering, University of Waterloo, 200 University \\ Avenue West, Waterloo, ON., N2L 3G1, Canada \\ Corresponding Author: \\ Lei Xu, University of Waterloo \\ 200 University Avenue West \\ Waterloo, ON. N2L 3G1 \\ Tel: (519) 888-4567 x36882 \\ Email: 1xu@uwaterloo.ca

\begin{abstract}
There are many cases where the elastic modulus of a structural member will vary longitudinally, such as when a steel column is heated by fire. In such a case, the fire can compromise the integrity of the structural frame. It is therefore necessary in stability analysis to accurately analyze members with longitudinally varying elastic modulus. A new analytical method is derived to evaluate the stability of an unbraced steel frame containing members that each consist of up to three segments of differing elastic modulus. The method is presented in the form of a lateral stiffness equation, which characterises the loss of stability in a frame when its lateral stiffness diminishes to zero. The proposed method is also demonstrated via a numerical example by analyzing the case of a post-blast explosion fire whereby the insulation along a segment of any member is damaged. A scenario analysis was also conducted to identify the most vulnerable location in a frame, whereby the damage to insulation resulting from a blast causes the greatest reduction to the fire resistance. From a design standpoint, the most vulnerable locations can be identified and further protected to improve safety. The proposed method provides more accurate assessments of frame stability in cases where elastic modulus vary longitudinally in members,
\end{abstract} \\ and is validated via finite element analysis.
}

Keywords: fire; stability; steel frame; unbraced; storey-based; segment; stepped; temperature distributions; semi-rigid; insulation damage 


\section{INTRODUCTION}

2 It is not uncommon for fires to occur in large buildings, especially those containing steel frames.

3 As a result, the effect of fires on structural steel frames has frequently been modeled numerically

4 in the past few decades [1-4]. However, due to the variable and unpredictable nature of fire,

5 assumptions will always be necessary in numerical models. Very often, these assumptions

6 include uniform member temperatures, such as in the cases of [1,5-6]. However, Xu and Zhuang

7 [3] demonstrated that the stability calculations of a steel frame can be significantly affected when

8 a two-stepped temperature distribution is modeled in its columns, rather than assuming uniform

9 temperature. The reason for using two-stepped columns is that room fires exhibit higher

10 temperatures near ceilings and lower temperatures near floors [3]. As such, the assumption of

11 two-stepped members is a progressive step towards realistic modelling of non-linear temperature

12 distributions in columns. Presented in this paper is a new methodology that evaluates the storey-

13 based lateral stability of an unbraced steel frame and extends the use of stepped members

14 towards applications where both columns and beams in a frame contain up to three segments of

15 differing temperatures. The presence of multiple segments of varying temperatures in members

16 of a frame can result from various fire scenarios, such as when fires initiate closer to one side of

17 a compartment, or when insulation is damaged during a post-earthquake or explosion fire.

18 Furthermore, an approach is presented for determining the individual buckling load three-stepped

19 column, which is the upper limit for the applicability of the lateral stiffness equation [7]. The

20 proposed method is demonstrated via numerical example whereby the damage to insulation due

21 to a blast explosion is modelled as a segment along any members of a frame. The heating of the

22 frame in fire until failure under various blast explosion scenarios is modelled to determine the

23 location in the frame whereby the blast results in the highest reduction to its fire resistance. 


\section{BACKGROUND}

25 The proposed method is in the form of a storey-based stability problem. The concept of storey-

26 based stability was initiated by Yura [8], who noted the fact that lateral instability could only

27 occur with all columns in the frame buckling simultaneously, and that structural frames perform

28 better when considered in whole over its individual members. Subsequently, LeMessurier [9],

29 Lui [10] and Aristizabal-Ochoa [11] have developed matrix methods for the storey stability analysis of steel frames. Xu [12] later derived the lateral stiffness equation for a frame subjected to axial loading with considering $P$ - $\Delta$ effects. Recently, Xu and Zhuang [3] extended Xu's method [12] to include members with elevated temperatures under fire, including columns containing two temperature zones along their lengths. However, the method proposed by $\mathrm{Xu}$ and Zhuang [3] does not apply for more complicated thermal distributions in the frame members, such as in the case of a large compartment containing a localized fire or travelling fire [4], or when considering the effects of insulation delamination at plastic hinges caused by seismic loading [13-16]. To account for all of these considerations, the proposed method is in the form of numerical procedures such as [5,17-18]. 
48 Consider the 2D storey frame with $n$ bays shown in Fig. 1. All frame members consist of three segments with different temperatures, assumed to be uniform within the segments. For columns,

50 the lower, middle, and upper segments are denoted by the primary subscripts $l, m$, and $u$,

51 respectively. For beams, the left, middle, and right segments are denoted by the primary

52 subscripts $L, M$, and $R$, respectively. The beams and bays are numbered with primary subscripts from 1 to $n$, and columns are numbered with secondary subscripts from 1 to $n+1$.

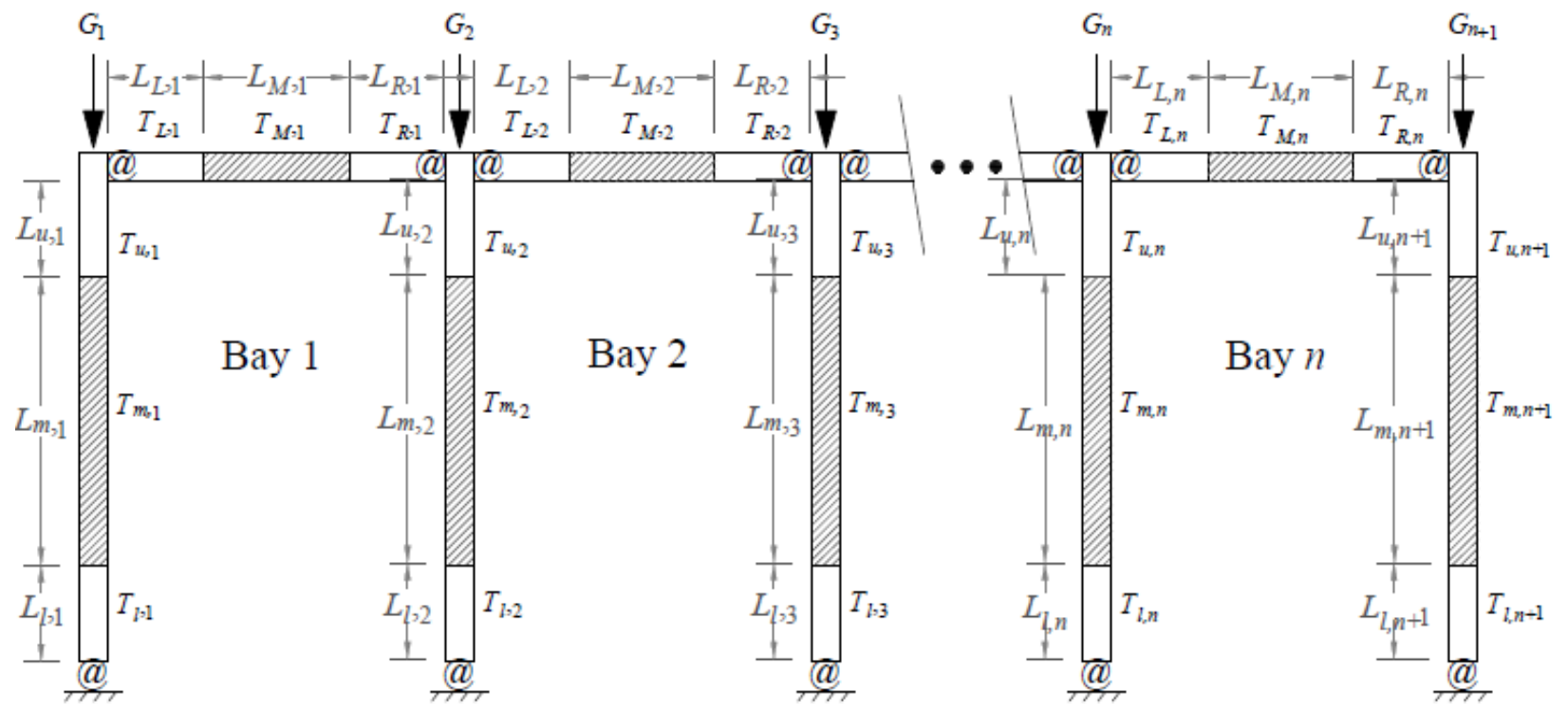

Figure 1 - Schematic of unbraced storey frame with three-segmented members

The temperatures and lengths of each member are denoted in Fig. 1 by the variables $T$ and $L$, respectively. Let the subscripts $i$ and $j$ correspond to the primary subscript of the columns and beams in the frame, respectively. $I_{c, i}$ and $I_{b, j}$ are the moments of inertia of column $i$ and beam $j$, respectively. The moment of inertia is assumed to be constant over the entire length of each member. Let $L_{c, i}=L_{u, i}+L_{m, i}+L_{l, i}$ be the height of column $i$, and $L_{b, j}=L_{L, j}+L_{M, j}+L_{R, j}$ be the length of beam $j$. The frame is subjected to prescribed gravity loads, $G_{i}$. The Eurocode 3 [19] method was adopted to model the degradation of the members due to elevated temperature, and 
63 considers the tangent modulus of elasticity as a function of the axial load, $P$, and temperature, $T$,

64 given in Eq. (1).

$$
E(P, T)=\mu_{T} E_{0} ; \quad \mu_{T}=\frac{1}{E_{0}}\left\{\begin{array}{cc}
E_{a}(T) & \sigma \leq f_{p}(T) \\
(b / a)\left(\varepsilon_{y}(T)-\varepsilon\right)\left[a^{2}-\left(\varepsilon_{y}(T)-\varepsilon\right)^{2}\right]^{-0.5}, f_{p}(T)<\sigma<f_{y}(T) & \\
0 & \sigma \geq f_{y}(T)
\end{array}\right.
$$

Where $E_{0}$ is the elastic modulus of the segment at ambient temperature and $\mu$ is the degradation factor for the elastic modulus. $\sigma$ and $\varepsilon$ are the stress and strain in the segment, respectively, and $a$ and $b$ are coefficients defined in Euroocde 3 [19]. $E_{a}, f_{p}$ and $f_{y}$ are the modulus in the linear elastic range, proportional limit and yield stress, respectively, also defined in Eurocode 3 [19] and are functions of the temperature. Note that the proposed methodology can be extended to account for non-fire scenarios, where $E_{0}$ can be any reference elastic modulus, and $\mu$ can be directly specified based on the relative elastic modulus in each segment.

\subsection{End Fixity Factors for Three-Segment Members}

All connections in the frame are generalized as semi-rigid connections. The end fixity factor concept established by Monforton and Wu [20] was employed to model the rotational stiffness of these connections. The end fixity factor, $r$, is defined as the ratio between the rotation at the end of the member, $\alpha$, and the combined rotation, $\phi$, of the member and the connection due to a unit end-moment, as shown in Fig. 2.

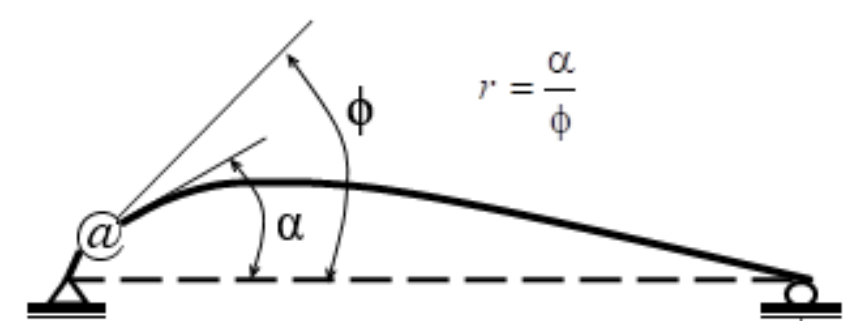

Figure 2 - Definition of End-fixity Factor

Let the upper and lower end fixity factors of column $i$ be denoted $r_{u, i}$ and $r_{l, i}$, respectively.

Similarly, let the end fixity factors at the corresponding ends of beam $j$ be $r_{L, j}$ and $r_{R, j}$, 
respectively. The end fixity factors for members with three segments of constant elastic modulus are derived in Appendix C and are given in Eqs. (2a) and (2b), respectively, for the columns and beams in the frame.

$$
\begin{gathered}
r_{l, i}=\frac{1}{1+\frac{3 E_{0} I_{c, i}}{L_{c} R_{l, i} \tau_{l, i}}} ; \quad r_{u, i}=\frac{1}{1+\frac{3 E_{0} I_{c, i}}{L_{c} R_{u, i} \tau_{u, i}}} \\
r_{L, j}=\frac{1}{1+\frac{3 E_{0} I_{b, j}}{L_{c} R_{L, j} \tau_{L, j}}} ; \quad r_{R, i}=\frac{1}{1+\frac{3 E_{0} I_{b, j}}{L_{c} R_{R, j} \tau_{R, j}}}
\end{gathered}
$$

Where $R$ is the rotational stiffness of the connection at the corresponding end subjected to elevated temperature and $\tau$ is an adjustment factor given in Eqs. (4) to account for the different temperatures in the segments. Note that for semi-rigid connections subjected to elevated temperature, $R$ can be adjusted via a reduction factor, $k_{R}$, in Eqs. (3) [21].

$$
\begin{gathered}
R\left(T_{R}\right)=k_{R} R_{0} \\
k_{R}=1-\frac{m}{R_{0}} T_{R} \geq 0
\end{gathered}
$$

Where $R_{0}$ is the rotational stiffness of the connection at ambient temperature and $T_{R}$ is the elevated temperature of the connection. For the purpose of simplicity, $T_{R}$ may be taken as the temperature of the nearest member segment. The value of $k_{R}$ can be obtained by correlations based on the experimental data in Al-Jabri et al. [22], whereby it is shown for various types of connections that the rotational stiffness parameter in the Ramberg-Osgood [23] rotational stiffness is linearly correlated with temperature. Based on the experimental results, the linear stiffness reduction slope factor, $m$, ranges between $1 \times 10^{4}$ and $6 \times 10^{4} \mathrm{Nm} /{ }^{\circ} \mathrm{C}$ [22]. Note that for idealized connections ( $R=0$ or $R=\infty$, corresponding to $r=0$ or $r=1$ ), the rotational stiffness is unaffected by temperature when using Eq. (3). 
108

The end fixity factors in Eqs. (2) differ from those derived by Monforton and $\mathrm{Wu}$ [20] in that they consider members with three segments via a $\tau$ factor, which accounts for the differences in temperatures in each segment and is defined in Eqs. (4a) and (4b) for columns and beams, respectively.

$$
\tau_{l, i}=\frac{1}{\mu_{l, i}}\left(\frac{L_{l, i}}{L_{c, i}}\right)^{3}+\frac{1}{\mu_{m, i}}\left(\left(\frac{L_{l, i}+L_{m, i}}{L_{c, i}}\right)^{3}+\left(\frac{L_{u, i}}{L_{c, i}}\right)^{3}\right)+\frac{1}{\mu_{u, i}}\left(1-\left(\frac{L_{l, i}+L_{m, i}}{L_{c, i}}\right)^{3}\right)
$$

$$
\begin{gathered}
\tau_{u, i}=\frac{1}{\mu_{u, i}}\left(\frac{L_{u, i}}{L_{c, i}}\right)^{3}+\frac{1}{\mu_{m, i}}\left(\left(\frac{L_{u, i}+L_{m, i}}{L_{c, i}}\right)^{3}+\left(\frac{L_{l, i}}{L_{c, i}}\right)^{3}\right)+\frac{1}{\mu_{l, i}}\left(1-\left(\frac{L_{u, i}+L_{m, i}}{L_{c, i}}\right)^{3}\right) \\
\tau_{L, j}=\frac{1}{\mu_{L, j}}\left(\frac{L_{L, j}}{L_{b, j}}\right)^{3}+\frac{1}{\mu_{M, i}}\left(\left(\frac{L_{L, j}+L_{M, j}}{L_{b, j}}\right)^{3}+\left(\frac{L_{R, j}}{L_{b, j}}\right)^{3}\right)+\frac{1}{\mu_{R, j}}\left(1-\left(\frac{L_{L, j}+L_{M, j}}{L_{b, j}}\right)^{3}\right)
\end{gathered}
$$

$$
\tau_{R, j}=\frac{1}{\mu_{R, j}}\left(\frac{L_{R, j}}{L_{b, j}}\right)^{3}+\frac{1}{\mu_{M, i}}\left(\left(\frac{L_{R, j}+L_{M, j}}{L_{b, j}}\right)^{3}+\left(\frac{L_{L, j}}{L_{b, j}}\right)^{3}\right)+\frac{1}{\mu_{L, j}}\left(1-\left(\frac{L_{R, j}+L_{M, j}}{L_{b, j}}\right)^{3}\right)
$$

Where $\mu$ is the degradation factor accounting for the effect of elevated temperatures on the elastic modulus in the corresponding segment of the member, obtained from Eq. (1). Note that Eqs. (4) extend the case of two-segmented members derived by $\mathrm{Xu}$ and Zhuang [3] to include three-segmented members. Therefore, the value of the end fixity factor varies based on the temperature of the segments of the members, as well as the axial load if the segment is in the non-linear elastic range. Note that $R=0$ for an idealized pinned connection, and $R=\infty$ for a fixed connection. Similarly, $r=0$ for a pinned connection, and $r=1$ for a fixed connection. Where multiple beams are connected to the end of column, $R$ is given as the sum of contributions from the beams in Eq. (5).

$$
R_{u, i}=\sum_{j_{u}=1}^{m_{u}} R_{i, j_{u}} ; \quad R_{l, i}=\sum_{j_{l}=1}^{m_{l}} R_{i, j_{l}}
$$


119 Where $R_{u, i}$ and $R_{l, i}$ are the rotational stiffness of the upper and lower end connections,

120 respectively. $m_{u}$ and $m_{l}$ are the number of beams connected to the upper and lower ends of

121 column $i$, respectively. The rotational resistance provided by beam $j$ to column $i$ at the

122 corresponding end, $R_{i, j}$, can be calculated using Eq. (6), with the corresponding derivation shown

123 in Appendix D.

$$
R_{i, j}=\frac{6 E_{0} I_{b, j} r_{N}}{L_{b, j}}\left[\frac{2 \tau_{F} \mu_{L} \mu_{M} \mu_{R}\left(1-r_{F}\right)+2 \lambda_{N N} r_{F}+\lambda_{N F} r_{F} v_{N F}}{4 \lambda_{A}+r_{N} \lambda_{B}+r_{F} \lambda_{C}-r_{N} r_{F} \lambda_{D}}\right]
$$

125

126

In which the subscript $N$ refers to the near end of beam $j$ connected to column $i$, and the subscript $F$ refers to the far end of beam $j$ connected to column $i$. These subscripts are to be replaced by the subscripts $L$ and $R$ as necessary. The values of $\mu_{N}, \mu_{M}$, and $\mu_{F}$ for the corresponding segments are obtained from Eq. (1), and $v_{N F}$ is the ratio between the near end and far end connection rotations and corresponds to the buckling shape which needs to be assumed in advance in order to simplify the problem for analytical solutions [24]. It was demonstrated by Xu \& Liu [24] that assuming $v_{N F}=1$ gives accurate estimations of results and corresponds to the asymmetric buckling mode. The coefficients $\lambda_{A}, \lambda_{B}, \lambda_{C}$, and $\lambda_{D}$ are given in Eqs. (7a) through (7d) and are defined such at $\lambda_{A}=\lambda_{D}=1$ and $\lambda_{B}=\lambda_{C}=0$ in the case of a single segment beam with uniform ambient temperature $\left(\mu_{N}=\mu_{M}=\mu_{F}=1\right)$. The coefficients $\lambda_{N N}$ and $\lambda_{N F}$ depend on the temperatures and lengths of each segment of the member, given in Eqs. (7e) and (7f).

$$
\begin{gathered}
\lambda_{A}=\mu_{L} \mu_{M} \mu_{R} \tau_{N} \tau_{F} \\
\lambda_{B}=4 \tau_{F}\left(\kappa_{N}-\tau_{N} \mu_{N} \mu_{M} \mu_{F}\right) \\
\lambda_{C}=4 \tau_{N}\left(\kappa_{F}-\tau_{F} \mu_{N} \mu_{M} \mu_{F}\right) \\
\lambda_{D}=4\left[\tau_{F} \kappa_{N}+\tau_{N} \kappa_{F}-\tau_{N} \tau_{F} \mu_{N} \mu_{M} \mu_{F}\right]-3 L_{b, j}^{4}\left(L_{N}^{4} \mu_{M} \mu_{F} / \mu_{N}+L_{M}^{4} \mu_{N} \mu_{F} / \mu_{M}+L_{F}^{4} \mu_{N} \mu_{M} / \mu_{F}+\ldots\right. \\
4 L_{N} L_{M}^{3} \mu_{F}+4 L_{N} L_{F}^{3} \mu_{M}+4 L_{M} L_{N}^{3} \mu_{F}+4 L_{M} L_{F}^{3} \mu_{N}+4 L_{F} L_{N}^{3} \mu_{M}+4 L_{F} L_{M}^{3} \mu_{N}+\ldots \\
\left.6 L_{N}^{2} L_{M}^{2} \mu_{F}+6 L_{N}^{2} L_{F}^{2} \mu_{M}+6 L_{M}^{2} L_{F}^{2} \mu_{N}+12 L_{N}^{2} L_{M} L_{F} \mu_{M}+12 L_{N} L_{M}^{2} L_{F} \mu_{M}+12 L_{N} L_{M} L_{F}^{2} \mu_{M}\right)
\end{gathered}
$$




$$
\begin{gathered}
\lambda_{N N}=\frac{1}{L_{b, j}{ }^{3}}\left(L_{N}^{3} \mu_{M} \mu_{F}+L_{M}^{3} \mu_{N} \mu_{F}+L_{F}^{3} \mu_{N} \mu_{M}+3 L_{N} L_{M}^{2} \mu_{N} \mu_{F}+3 L_{N} L_{F}^{2} \mu_{N} \mu_{M}+3 L_{M} L_{N}^{2} \mu_{N} \mu_{F}+\ldots\right. \\
\left.\quad 3 L_{F} L_{M}^{2} \mu_{N} \mu_{F}+3 L_{F} L_{N}^{2} \mu_{F} \mu_{M}+3 L_{M} L_{F}^{2} \mu_{M} \mu_{N}+6 L_{N} L_{M} L_{F} \mu_{N} \mu_{M}\right) \\
\lambda_{N F}=\frac{1}{L_{b, j}{ }^{3}}\left(L_{N}^{3} \mu_{M} \mu_{F}+L_{M}^{3} \mu_{N} \mu_{F}+L_{F}^{3} \mu_{N} \mu_{M}+3 L_{N} L_{M}^{2} \mu_{N} \mu_{F}+3 L_{N} L_{F}^{2} \mu_{N} \mu_{M}+3 L_{M} L_{N}^{2} \mu_{M} \mu_{F}+\ldots\right. \\
\left.3 L_{F} L_{M}^{2} \mu_{N} \mu_{F}+3 L_{F} L_{N}^{2} \mu_{F} \mu_{M}+3 L_{M} L_{F}^{2} \mu_{N} \mu_{M}+6 L_{N} L_{M} L_{F} \mu_{N} \mu_{F}\right)
\end{gathered}
$$

143 Where the coefficients $\kappa_{N}$ and $\kappa_{F}$ also depend on the temperatures and lengths of each segment of

144 the member and affect the coefficients $\lambda_{B}, \lambda_{C}$ and $\lambda_{D}$, given in Eqs. (8).

$$
\begin{aligned}
& \kappa_{N}=\frac{1}{L_{b, j}^{4}}\left[\left(L_{N}^{4} \mu_{M} \mu_{F}+L_{M}^{4} \mu_{N} \mu_{F}+L_{F}^{4} \mu_{N} \mu_{M}\right)+\ldots\right. \\
& 3\left(L_{N} L_{M}^{3} \mu_{M} \mu_{F}+L_{N} L_{F}^{3} \mu_{M} \mu_{F}+L_{M} L_{F}^{3} \mu_{N} \mu_{F}\right)+\ldots \\
& 4\left(L_{M} L_{N}^{3} \mu_{M} \mu_{F}+L_{F} L_{N}^{3} \mu_{M} \mu_{F}+L_{F} L_{M}^{3} \mu_{N} \mu_{F}\right)+\ldots \\
& \left(L_{N} L_{M}^{3} \mu_{N} \mu_{F}+L_{N} L_{F}^{3} \mu_{N} \mu_{M}+L_{M} L_{F}^{3} \mu_{N} \mu_{M}\right)+\ldots \\
& 6\left(L_{N}^{2} L_{M}^{2} \mu_{M} \mu_{F}+L_{N}^{2} L_{F}^{2} \mu_{M} \mu_{F}+L_{M}^{2} L_{F}^{2} \mu_{N} \mu_{F}\right)+\ldots \\
& 9\left(L_{N} L_{M}^{2} L_{F} \mu_{M} \mu_{F}+L_{N} L_{M} L_{F}^{2} \mu_{M} \mu_{F}\right)+\ldots \\
& 12\left(L_{N}^{2} L_{M} L_{F} \mu_{M} \mu_{F}\right)+\ldots \\
& \left.3\left(L_{N} L_{M}^{2} L_{F} \mu_{N} \mu_{F}+L_{N} L_{M} L_{F}^{2} \mu_{N} \mu_{F}\right)\right] \\
& \kappa_{F}=\frac{1}{L_{b, j}^{4}}\left[\left(L_{N}^{4} \mu_{M} \mu_{F}+L_{M}^{4} \mu_{N} \mu_{F}+L_{F}^{4} \mu_{N} \mu_{M}\right)+\ldots\right. \\
& 3\left(L_{M} L_{N}^{3} \mu_{N} \mu_{F}+L_{F} L_{N}^{3} \mu_{N} \mu_{M}+L_{F} L_{M}^{3} \mu_{N} \mu_{M}\right)+\ldots \\
& 4\left(L_{N} L_{M}^{3} \mu_{N} \mu_{F}+L_{N} L_{F}^{3} \mu_{N} \mu_{M}+L_{M} L_{F}^{3} \mu_{N} \mu_{M}\right)+\ldots \\
& \left(L_{M} L_{N}^{3} \mu_{M} \mu_{F}+L_{F} L_{N}^{3} \mu_{M} \mu_{F}+L_{F} L_{M}^{3} \mu_{N} \mu_{F}\right)+\ldots \\
& 6\left(L_{N}^{2} L_{M}^{2} \mu_{N} \mu_{F}+L_{N}^{2} L_{F}^{2} \mu_{N} \mu_{F}+L_{M}^{2} L_{F}^{2} \mu_{N} \mu_{M}\right)+\ldots \\
& 9\left(L_{N} L_{M}^{2} L_{F} \mu_{N} \mu_{M}+L_{F} L_{M} L_{N}^{2} \mu_{M} \mu_{N}\right)+\ldots \\
& 12\left(L_{F}^{2} L_{M} L_{N} \mu_{M} \mu_{N}\right)+\ldots \\
& \left.3\left(L_{N} L_{M}^{2} L_{F} \mu_{N} \mu_{F}+L_{F} L_{M} L_{N}^{2} \mu_{N} \mu_{F}\right)\right]
\end{aligned}
$$

147 Note that for single segment beams under uniform ambient temperature conditions $\left(\mu_{N}=\mu_{M}=\mu_{F}\right.$

$148=1$ ), Eq. (6) converges to the equation for $R_{i, j}$ derived by Monforton and Wu [20]. Where no

149 other members contribute to the rotational rigidity of the end connection of a member, the end

150 fixity factor at the corresponding end may be calculated using Eqs. (3). Expressing this in terms

151 of the end fixity factor at ambient temperature gives Eq. (9) below. 


$$
r=\frac{r_{0} k_{R} \tau}{1-r_{0}\left(1-k_{R} \tau\right)}
$$

153 Where $r$ is the end fixity factor at the condition-specified end, $\tau$ is the corresponding factor in Eqs.

154 (4), and $r_{0}$ is the end fixity factor of the corresponding end under ambient temperature conditions.

155 Note that in the cases of pinned connections $\left(r_{0}=0\right)$ and fixed connections $\left(r_{0}=1\right)$ at ambient

156 temperatures, the resulting end fixity factors at elevated temperatures remain unchanged $\left(r=r_{0}\right)$.

\section{$157 \quad 3.2 \quad$ Thermal Restraints}

158 The total axial load of column $i$ experiencing elevated temperatures can be expressed as $P_{i}=G_{i}$

$159+H_{i}$, with $G_{i}$ being the applied gravity load and $H_{i}$ being the additional axial load induced by

160 restraint against thermal strains. $H_{i}$ can be taken as zero where there are no physical restraints

161 against thermal strains, or calculated using the procedure in Appendix A for restrained columns.

\section{$162 \quad 3.3 \quad$ Storey-based Lateral Stiffness}

163 In the proposed method, the storey-based lateral stiffness of the frame in Fig. 1 is calculated to

164 evaluate the frame's stability. The lateral stiffness of the frame is its ability to resist lateral

165 deformation under given loading conditions, and is defined as the lateral force required to cause

166 a unit lateral displacement of the storey. The frame becomes unstable when the lateral stiffness

167 of the storey reaches zero [3,12].

168 In order to evaluate the lateral stiffness of an unbraced frame with three-segmented members, the

169 lateral stiffness of a single three-segmented column illustrated in Fig. 3 must first be derived. For

170 purposes of clarity, the subscript $i$ is removed from Fig. 3 and subsequent equations referring to

171 the variables and properties of this individual column. In order to account for $P-\Delta$ effects, the

172 axial load $P$ is also applied to the column. 


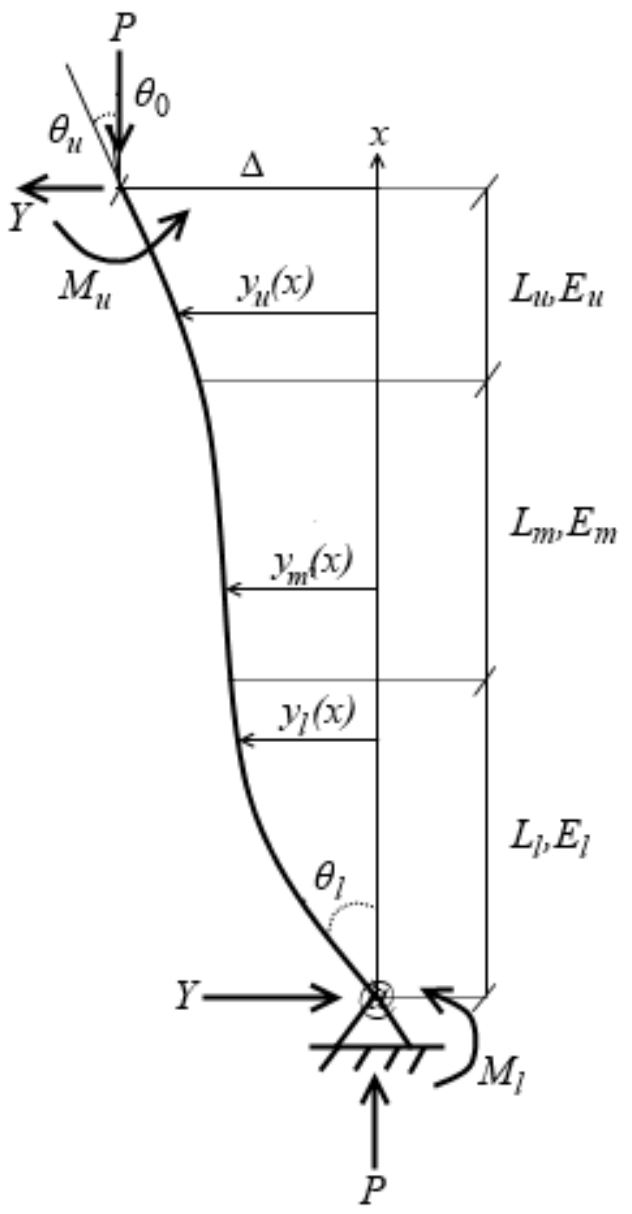

175 A lateral load, $Y$, is assumed to act at each end of the column. The transverse deflection

176 coordinates $y_{l}(x), y_{m}(x)$, and $y_{u}(x)$ apply to the corresponding segments of the member. The

177 rotational springs at each end of the column produce end moments $M_{u}$ and $M_{l}$ as per Eqs. (10).

$$
\begin{aligned}
M_{u} & =R_{u} \theta_{u} \\
M_{l} & =R_{l} \theta_{l}
\end{aligned}
$$

180 Where the rotational stiffnesses of the upper and lower connections, $R_{u}$ and $R_{l}$, respectively, can 181 be obtained from Eqs. (3). Based on external equilibrium, Eq. (11) must be satisfied.

$$
M_{u}+M_{l}=Y L_{c}+P \Delta
$$

183 Based on internal equilibrium via the method of sections, the internal bending moments in the 184 three segments of the column are given in Eqs. (12). 


$$
E_{l} I_{c} \frac{d^{2} y_{l}}{d x^{2}}=M_{l}-P\left(y_{l}(x)\right)-Y x ; \quad 0 \leq x \leq L_{l}
$$

$$
E_{l} I_{c} \frac{d^{2} y_{m}}{d x^{2}}=M_{l}-P\left(y_{m}(x)\right)-Y x ; \quad L_{l} \leq x \leq L_{l}+L_{m}
$$

$$
E_{l} I_{c} \frac{d^{2} y_{u}}{d x^{2}}=M_{l}-P\left(y_{u}(x)\right)-Y x ; \quad L_{l}+L_{m} \leq x \leq L_{c}
$$

The system of differential equations in Eqs. (12) can be solved by applying the eight boundary and compatibility conditions in Eqs. (13), in addition to the external moment equation in Eq. (10).

$$
\begin{gathered}
y_{l}(0)=0 \\
y_{u}\left(L_{c}\right)=\Delta \\
y^{\prime}{ }_{l}(0)=\theta_{l} \\
y^{\prime}{ }_{u}\left(L_{c}\right)=\theta_{u} \\
y_{l}\left(L_{l}\right)=y_{m}\left(L_{l}\right) \\
y_{m}\left(L_{l}+L_{m}\right)=y_{u}\left(L_{l}+L_{m}\right) \\
y_{l}^{\prime}\left(L_{l}\right)=y_{m}^{\prime}\left(L_{l}\right) \\
y_{m}^{\prime}\left(L_{l}+L_{m}\right)=y^{\prime}{ }_{u}\left(L_{l}+L_{m}\right)
\end{gathered}
$$

Eqs. (13a) through (13d) are boundary conditions at the ends of the column, whereas Eqs. (13e) through (13h) are compatibility conditions that define deformation continuity between each segment of the column. Based on the solution to the system of differential equations in Eqs. (12), the lateral stiffness of the column is equal to $Y / \Delta$, which is expressed in Eqs. (14).

$$
\begin{gathered}
\frac{Y}{\Delta}=\frac{\phi^{2} E_{0} I_{c}}{L_{c}{ }^{3}}\left(\frac{1}{\psi-1}\right) \\
\psi=\frac{9 r_{u} r_{l} a_{5}-3 \phi^{2}\left[r_{l}\left(1-r_{u}\right) \tau_{u}+r_{u}\left(1-r_{l}\right) \tau_{l}\right] a_{1}}{\phi^{4} \tau_{l} \tau_{u}\left(1-r_{l}\right)\left(1-r_{u}\right) a_{1}+9 r_{u} r_{l} a_{2}-3 \phi^{2}\left[r_{l}\left(1-r_{u}\right) \tau_{u} a_{3}+r_{u}\left(1-r_{l}\right) \tau_{l} a_{4}\right]}
\end{gathered}
$$

Where the coefficients $a_{1}$ through $a_{5}$ are given in Eqs. (15).

$$
\begin{gathered}
a_{1}=\phi_{m}^{2} S_{l} S_{m} S_{u}-\phi_{l} \phi_{m} C_{l} C_{m} S_{u}-\phi_{m} \phi_{u} S_{l} C_{m} C_{u}-\phi_{l} \phi_{u} C_{l} S_{m} C_{u} \\
a_{2}=\phi_{l}^{2} \phi_{m} \phi_{u} S_{l} C_{m} C_{u}+\phi_{l} \phi_{m}^{2} \phi_{u} C_{l} S_{m} C_{u}+\phi_{l} \phi_{m} \phi_{u}^{2} C_{l} C_{m} S_{u}-\phi_{l}^{2} \phi_{u}^{2} S_{l} S_{m} S_{u} \\
a_{3}=\phi_{l}^{2} \phi_{m} S_{l} C_{m} S_{u}+\phi_{l} \phi_{m}^{2} C_{l} S_{m} S_{u}+\phi_{l}^{2} \phi_{u} S_{l} S_{m} C_{u}-\phi_{l} \phi_{m} \phi_{u} C_{l} C_{m} C_{u} \\
a_{4}=\phi_{l} \phi_{u}^{2} C_{l} S_{m} S_{u}+\phi_{m} \phi_{u}^{2} S_{l} C_{m} S_{u}+\phi_{m}^{2} \phi_{u} S_{l} S_{m} C_{u}-\phi_{l} \phi_{m} \phi_{u} C_{l} C_{m} C_{u} \\
a_{5}=a_{3}+a_{4}+2 \phi_{l} \phi_{m} \phi_{u}
\end{gathered}
$$


210 in which the modified loading coefficients $\phi_{l}, \phi_{m}$ and $\phi_{u}$ are related to the axial load factor of the

211 column, $\phi$, and modified by the degradation factor $\mu$ of the corresponding segment due to

212 elevated temperature. $S_{l}, S_{m}, S_{u}, C_{l}, C_{m}$ and $C_{u}$ are all trigonometric functions of the

213 corresponding modified load coefficients associated with each segment of the member. These

214 functions are given in Eqs. (16).

$$
\begin{gathered}
\phi=\sqrt{\frac{P}{E_{0} I_{c}}} L_{c} ; \quad \phi_{l}=\frac{\phi}{\sqrt{\mu_{l}}} ; \quad \phi_{m}=\frac{\phi}{\sqrt{\mu_{m}}} ; \quad \phi_{u}=\frac{\phi}{\sqrt{\mu_{u}}} \\
S_{l}=\sin \left(\frac{\phi_{l} L_{l}}{L_{c}}\right) ; \quad S_{m}=\sin \left(\frac{\phi_{m} L_{m}}{L_{c}}\right) ; \quad S_{u}=\sin \left(\frac{\phi_{u} L_{u}}{L_{c}}\right) \\
C_{l}=\cos \left(\frac{\phi_{l} L_{l}}{L_{c}}\right) ; \quad C_{m}=\cos \left(\frac{\phi_{m} L_{m}}{L_{c}}\right) ; \quad C_{u}=\cos \left(\frac{\phi_{u} L_{u}}{L_{c}}\right)
\end{gathered}
$$

218 Assuming that all of the columns of the storey experience the same deflection, the lateral

219 stiffness of a storey in the frame is the sum of the contributions of the individual columns, given

220 in Eq. (17).

$$
\Sigma S=\sum_{i=1}^{n+1} \frac{Y_{i}}{\Delta}=\sum_{i=1}^{n+1}\left[\frac{\phi_{i}{ }^{2} E_{0} I_{c, i}}{L_{c, i}{ }^{3}}\left(\frac{1}{\psi_{i}-1}\right)\right]
$$

222 This assumption is valid where rigid floor systems are provided to render the beams inextensible.

223 Eq. (17) is applicable as long as $P_{i}$ is positive and does not exceed the critical load of the

224 individual column, $P_{u, i}$, at which yielding of the section or rotational buckling occurs, shown in

225 Eq. (18).

$$
0 \leq P_{i} \leq P_{u, i}=\min \left\{P_{b, i}, P_{y, i}\right\}
$$

227 Where $P_{y, i}$ is the yielding load of the column equal to the product of the section area and the

228 lowest yield stress in the column, and $P_{b, i}$ is the rotational buckling load determined via 229 Appendix B. 
230 Note that the proposed method applies to one-storey frames. However, it can be extended for use

231 in multi-storey frames either via the storey decomposition method proposed by Liu and $\mathrm{Xu}$ [25],

232 whereby a multi-storey frame can be decomposed into individual stories for analysis using the

233 storey-based stability approach. The equivalent rotational stiffness of the column connections

234 can be computed at each storey level, and instability is defined to occur when the product sum of

235 the lateral stiffness in each storey diminishes to zero.

\section{$236 \quad 3.4 \quad$ Modelling of Nonlinear Temperature Distribution}

237 The proposed model using three-segment members can be applied towards different structural 238 engineering applications. Most generally, if non-linear temperature distributions occur in the

239 members, such temperature distributions can be represented using three segments in the

240 proposed method, each with their own average temperatures. Non-linear temperature

241 distributions may result from localized fires, such as when a fire is located in a corner of a room,

242 causing heating at ends of beams. Also, since warm air rises, vertical gradients of temperatures

243 are commonly observed in room fires [3]. Finally, yielding can occur near connections during

244 seismic loading, causing localized loss of fire protection [14-16] and resulting in higher

245 temperatures in the cross sections located in these areas during a post-earthquake fire. In the

246 proposed method, the lateral stiffness of the frame can be calculated if the temperatures of every

247 segment in each member are directly specified. This can be accomplished using any thermal

248 analysis methods, such as the incremental time step method proposed by Pettersson et al. [17], or

249 from finite element analysis. For example, Arablouei and Kodur [14] simulated the effects of

250 localized insulation damage on temperatures in members segments by determining the relative

251 temperatures between insulated and exposed steel obtained from finite element analysis.

252 Relationships between the temperatures of different segments within the frame can therefore be 
253 predicted with respect to the fire or reference temperature. In other words, the temperature of a

254 given segment may be taken as a function of either a reference temperature or the duration of fire.

$255 \quad 3.5 \quad$ Computational Procedure

256 A summary of the procedure that can be followed to analyze the storey-based stability of frames

257 with three-segmented members subjected to fire conditions using the proposed method is

258 provided below.

259 1. Specify the lengths of the segments in each member $\left(L_{l}, L_{m}, L_{u}, L_{L}, L_{M}, L_{R}\right)$. Determine

260 other member properties $\left(I_{c}, I_{b}\right)$ and specify $v_{N F}=1$ as necessary.

2612 . Input the temperatures, $T_{k}$, of each segment in each member

262 3. Input the specified gravity loads, $G_{i}$ and calculate the thermal restraint forces, $H_{i}$, where 263 applicable according to the procedure in Appendix A.

264 4. Calculate the resulting degradation factors and elastic modulus $(\mu$ and $E)$ using the Eurocode 3 [19] method in Eq. (1). Alternatively, $E_{0}$ and $\mu$ can be specified manually.

5. Calculate the end fixity factors for all ends of all members $\left(r_{u}, r_{l}, r_{N}, r_{F}\right)$ using Eqs. (3). Note that for member ends not dependent on the rotational resistance of other members, $r$ must be adjusted due to elevated temperature according to Eq. (9).

6. Ensure that the values of $P_{i}$ do not exceed $P_{u, i}$ in Eq. (18). If $P_{u, i}$ is exceeded then the column has failed locally via rotational buckling or yielding.

7. Calculate lateral stiffness contribution, $S_{i}$, for each column. The lateral stiffness, $\Sigma S$, is the summation of the lateral stiffness contribution from all columns in the storey frame in Eq. (17). If $\Sigma S>0$ then the frame is stable. Instability analysis can also be performed by increasing either the applied gravity loads or temperatures of the members until instability occurs $(\Sigma S=0)$. 
277 A numerical example is provided to demonstrate the use of the proposed method towards a frame

278 with three-segmented members in post-explosion fire scenarios. Explosions in buildings can

279 cause local damages to insulation on members, and the lengths of the regions of damage can be 280 modelled as segments of the members [14]. Moreover, explosions can ignite nearby fuel and 281 cause room fires. Consider the two-bay frame shown in Fig. 4, where the sensitivity of the frame 282 lateral stiffness to the location of insulation damage due to explosion blasts is analyzed.

283

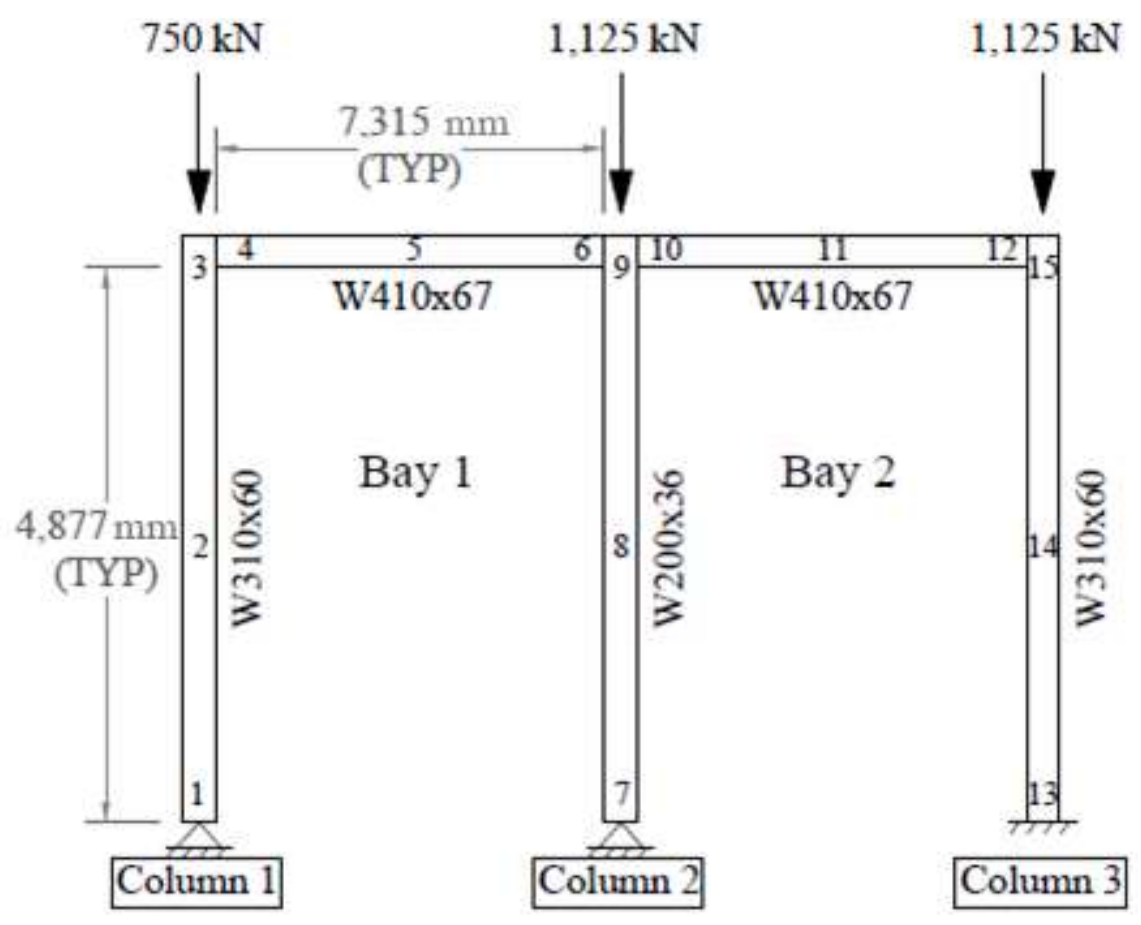

Figure 4 - Example two-bay unbraced storey frame subjected to post-explosion fire

In each scenario, a blast is assumed to cause local delamination to a $1.0 \mathrm{~m}$ long segment at either an end or the middle of a member, followed by an ASTM E119 [26] fire, assumed to occur uniformly throughout the entire frame. The member subjected to insulation damage at an end or in the middle can conveniently be modelled as a two- or three-segment member, respectively. Note that single- and two-segment members are modelled as three-segment members with 
290 identical properties in adjacent segments. The blast locations are numbered in Fig. 4 for each

291 scenario (1 to 15). The scenarios are also compared to the case of a completely undamaged frame.

292 The original thickness of insulation required to provide a nominal fire resistance of $R_{N}=120$

293 minutes based on the prescriptive approach in Eq. (19) is applied on each member [27].

$$
t_{p}=\frac{25.4 R_{N}}{1.03 W / D+42}
$$

295 Where $t_{p}$ is the thickness of the protective insulation $(\mathrm{mm})$ required to provide the desired fire 296 resistance rating, $R(\mathrm{~min})$, for a steel member with unit weight $W(\mathrm{~kg} / \mathrm{m})$ and heated perimeter $D$ 297 (m). The density, thermal conductivity and heat capacity of insulation are assumed to be 400 $\mathrm{kg} / \mathrm{m}^{3}, 0.12 \mathrm{~W} / \mathrm{mK}$ and $1,500 \mathrm{~J} / \mathrm{kgK}$, respectively. The section properties are tabulated in Table 1.

Table 1 - Member section properties in two-bay frame example

\begin{tabular}{cccccc}
\hline Property & $I$ & $A$ & $D$ & $W$ & $t_{p}$ \\
\hline W200x36 & $34.1 \times 10^{6} \mathrm{~mm}^{4}$ & $4,570 \mathrm{~mm}^{2}$ & $1.05 \mathrm{~m}$ & $36 \mathrm{~kg} / \mathrm{m}$ & $39.4 \mathrm{~mm}$ \\
W310x60 & $129 \times 10^{6} \mathrm{~mm}^{4}$ & $7,610 \mathrm{~mm}^{2}$ & $1.40 \mathrm{~m}$ & $60 \mathrm{~kg} / \mathrm{m}$ & $35.4 \mathrm{~mm}$ \\
W410x67 & $245 \times 10^{6} \mathrm{~mm}^{4}$ & $8,580 \mathrm{~mm}^{2}$ & $1.52 \mathrm{~m}$ & $67 \mathrm{~kg} / \mathrm{m}$ & $34.9 \mathrm{~mm}$ \\
\hline
\end{tabular}

301 The time-temperature relationships for the segments in each member subjected to the ASTM fire 302 were computed using a 2D heat transfer finite element model in ABAQUS. The gas temperature 303 is assumed to be uniform throughout the frame. Within the $1.0 \mathrm{~m}$ delamination length in each 304 scenario, the insulation on one flange of the section is assumed to be removed. The density, 305 thermal conductivity and heat capacity of steel are assumed to be $7,850 \mathrm{~kg} / \mathrm{m}^{3}, 40 \mathrm{~W} / \mathrm{mK}$ and 306 $600 \mathrm{~J} / \mathrm{kgK}$, respectively. A convective heat transfer coefficient of $h=25 \mathrm{~W} / \mathrm{m}^{2} \mathrm{~K}$ and emissivity 307 of 0.9 was assumed for all exposed surfaces. Quadratic heat transfer elements were used in the 308 section meshes. As the blast damage can occur on any one of segment of the members in the 309 frame, all of the sections in Table 1 are illustrated in Fig. 5 as protected with either damaged 310 insulation (DI) and undamaged insulation (UI). 


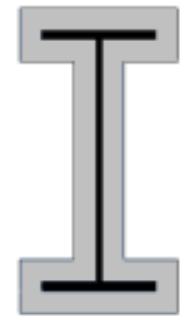

W410 (UI)

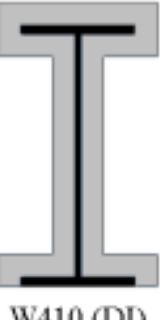

W410 (DI)

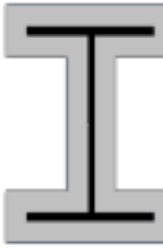

W310 (UI)

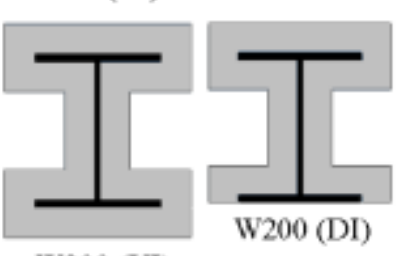

W200 (UI)

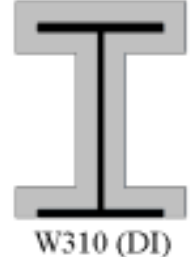

313 The cross-sectional temperatures in the member segments under the these cases are plotted

314 versus the duration and gas temperature of the ASTM E119 [26] fire in Fig. 6.

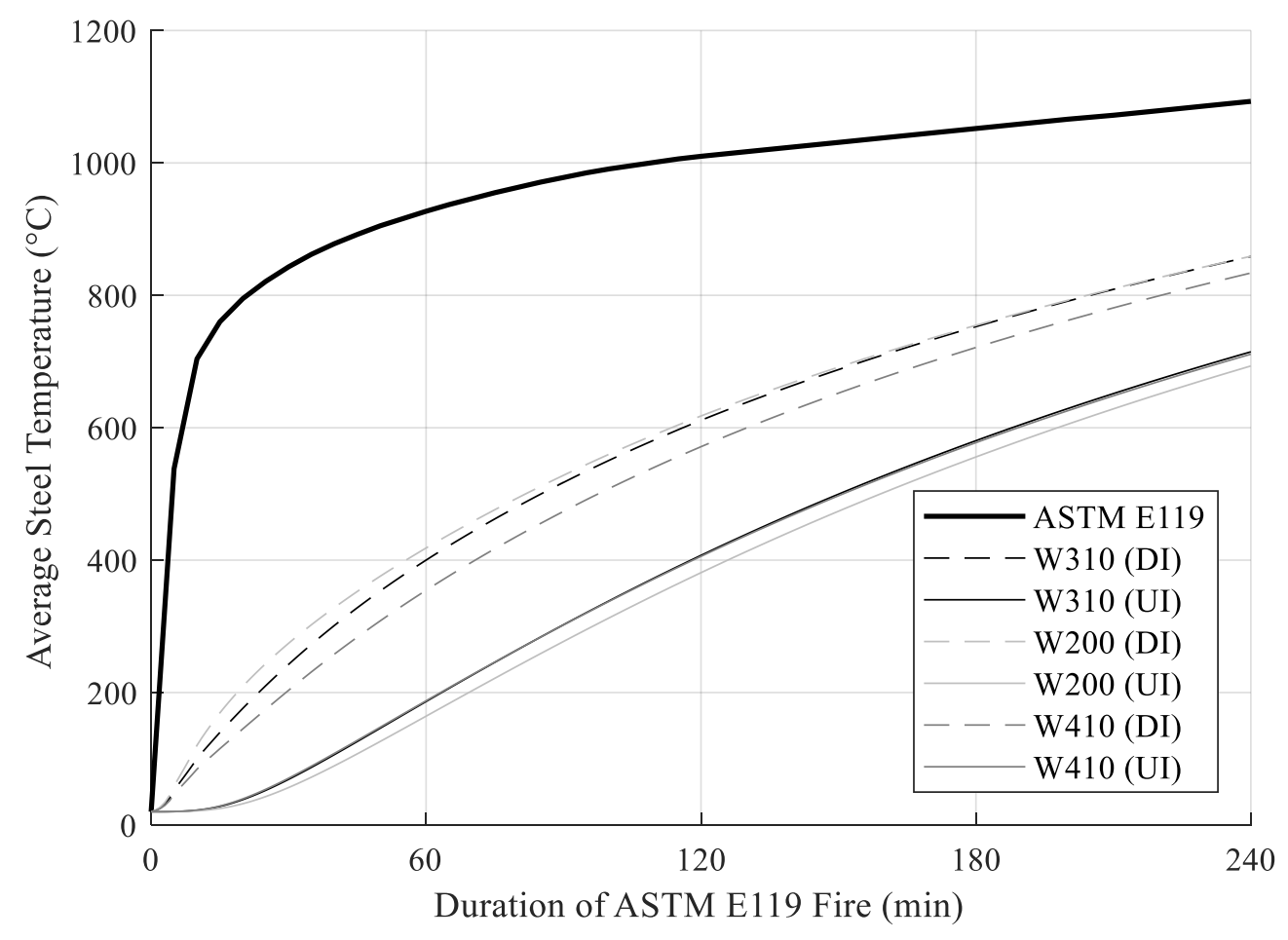

Figure 6 - Time-temperature results from finite element analysis of segment cross-sections

317 From Fig. (6), it is observed that when any section is subjected to damaged insulation its

318 temperature is increased by up to $255^{\circ} \mathrm{C}$ over the course of the fire event compared to when it is 
not damaged. The frame in Fig. 4 is subjected to the prescribed gravity loads shown, which are

320 constant in the analysis. However, the columns are thermally restrained, and the additional axial

321 forces in columns due to thermal expansion will be calculated according to Appendix A. As such,

322 the internal axial forces, $P_{i}$, in the columns increase as the temperatures increase. Column 3 is

323 rigidly connected to the ground $\left(r_{l, 3}=1\right)$ while the other columns are pinned to the ground $\left(r_{l, 1}=\right.$

$\left.324 r_{l, 2}=0\right)$. The ambient modulus of elasticity is $E_{0}=200 \mathrm{GPa}$. All beam-to-column connections are

325 assumed to be semi-rigid end plate connections, with $r_{N, 0}=r_{F, 0}=0.493$ for all beams in Eq. (8),

326 which corresponds to ambient rotational stiffnesses of $R_{0}=19.56 \times 10^{6} \mathrm{Nm}$. The linear stiffness

327 reduction slope factor is taken as $m=2.88 \times 10^{4} \mathrm{Nm} /{ }^{\circ} \mathrm{C}$. The $R_{0}$ and $m$ parameters were selected

328 based on a linear regression analysis of the results of Al-Jabri et al. [22] for Group 2 end plate

329 connections. The coefficient of determination for fitting the experimental data with the selected

330 parameters was $R^{2}=0.97$. As the duration of fire is increased, the lateral stiffness of the frame

331 subjected to blast damages diminishes. The duration of fire at which lateral instability failure of

332 the frame occurs, along with the maximum value of $P_{i} / P_{u, i}$ at the time of failure, is listed for each

333 of the scenarios in Table 2, where $P_{u, i}$ is the rotational buckling load that varies with temperature.

334 The failure times corresponding to two analyses are reported in Table 2: (1) with assuming

335 asymmetrical buckling $\left(v_{N F}=1\right)$ as necessary in the proposed method, and (2) with values of $v_{N F}$

336 calibrated at each beam-to-column connection based on an eigenvalue buckling analysis

337 conducted in ABAQUS.

Table 2 - Failure durations of fire in scenario analysis of frame subjected to post-explosion fires

\begin{tabular}{ccccc}
\hline Scenario & Damaged Location & $\begin{array}{c}\text { Failure Time } \\
\left(v_{N F}=1\right)\end{array}$ & $\begin{array}{c}\text { Failure Time } \\
\left(v_{N F} \text { calibrated }\right)\end{array}$ & $\left(P_{i} / P_{u, i}\right)_{\max }$ \\
\hline $\mathrm{U}$ & Undamaged frame & $138.8 \mathrm{~min}$ & $136.2 \mathrm{~min}$ & $P_{2} / P_{u, 2}=0.90$ \\
1 & Column 1, lower end & $136.7 \mathrm{~min}$ & $134.9 \mathrm{~min}$ & $P_{2} / P_{u, 2}=0.89$ \\
2 & Column 1, middle & $129.9 \mathrm{~min}$ & $128.8 \mathrm{~min}$ & $P_{1} / P_{u, 1}=0.85$ \\
3 & Column 1, upper end & $131.8 \mathrm{~min}$ & $130.2 \mathrm{~min}$ & $P_{1} / P_{u, 1}=0.86$ \\
4 & Beam 1, left end & $134.7 \mathrm{~min}$ & $132.5 \mathrm{~min}$ & $P_{2} / P_{u, 2}=0.87$
\end{tabular}




\begin{tabular}{ccccc}
5 & Beam 1, middle & $138.8 \mathrm{~min}$ & $135.9 \mathrm{~min}$ & $P_{2} / P_{u, 2}=0.90$ \\
6 & Beam 1, right end & $138.4 \mathrm{~min}$ & $136.3 \mathrm{~min}$ & $P_{2} / P_{u, 2}=0.90$ \\
7 & Column 2, lower end & $101.4 \mathrm{~min}$ & $101.0 \mathrm{~min}$ & $P_{2} / P_{u, 2}=0.99$ \\
8 & Column 2, middle & $107.0 \mathrm{~min}$ & $104.6 \mathrm{~min}$ & $P_{2} / P_{u, 2}=0.97$ \\
9 & Column 2, upper end & $109.7 \mathrm{~min}$ & $109.7 \mathrm{~min}$ & $P_{2} / P_{u, 2}=1.00^{*}$ \\
10 & Beam 2, left end & $138.1 \mathrm{~min}$ & $136.5 \mathrm{~min}$ & $P_{2} / P_{u, 2}=0.90$ \\
11 & Beam 2, middle & $138.8 \mathrm{~min}$ & $135.8 \mathrm{~min}$ & $P_{2} / P_{u, 2}=0.90$ \\
12 & Beam 2, right end & $130.6 \mathrm{~min}$ & $128.6 \mathrm{~min}$ & $P_{2} / P_{u, 2}=0.85$ \\
13 & Column 3, lower end & $107.6 \mathrm{~min}$ & $103.7 \mathrm{~min}$ & $P_{3} / P_{u, 3}=0.78$ \\
14 & Column 3, middle & $121.0 \mathrm{~min}$ & $120.4 \mathrm{~min}$ & $P_{3} / P_{u, 3}=0.98$ \\
15 & Column 3, upper end & $120.9 \mathrm{~min}$ & $120.7 \mathrm{~min}$ & $P_{3} / P_{u, 3}=0.98$ \\
\hline \multicolumn{5}{c}{$*$ Denotes a value that is slightly below but rounds to unity }
\end{tabular}

340 To obtain the calibrated values for each scenario, the frame in Fig. 4 was modelled in ABAQUS

341 by using B23 cubic Euler-Bernoulli (non-shear-deformable) wireframe elements in all members.

342 The semi-rigid connections were also modelled using linear-elastic "Join + Rotation" connector

343 section features, with temperature-dependent values of $R$. In the eigenvalue buckling analysis,

344 the loads were proportionally assigned. The thermal restraints were considered by applying an

345 additional factor to the gravity loads equal to the proportional increase in axial load experienced

346 by the column due to the thermal restraints as calculated via the procedure in Appendix A. The

347 time of failure corresponding to the calibrated $v_{F N}$ values in Table 2 were obtained via trial and

348 error in changing the elastic modulus of the segments based on Eq. (1) and repeating the

349 eigenvalue buckling analysis in ABAQUS until the critical applied gravity load factor of the

350 frame corresponded exactly to the given applied loads. The calibrated values of $v_{N F}$ were then

351 retrieved from the FEA model based on the rotational displacements from the buckled shape of

352 the frame at the time of failure in ABAQUS. These values were inputted to the proposed method

353 for re-analysis, and the failure times as determined using the proposed method with the

354 calibrated values are presented in the corresponding column of Table 2 . The resulting time of

355 failure differed by at most only 0.02 minutes $(0.015 \%)$ between the proposed method and the

356 FEA model out of all the scenarios. Further to this, the time of failure obtained using the 
357 calibrated $v_{F N}$ values in the proposed method were inputted into ABAQUS whereby the resulting 358 critical load factor was calculated. The proportional load factors applied in ABAQUS were 1, 1 359 and 1.5, corresponding to Columns 1, 2 and 3, respectively. As such, a critical load factor of 750 $360 \mathrm{kN}$ would correspond to zero error between the FEA model and the proposed method. Out of all 361 of the scenarios, the largest error in the critical load factor calculated in ABAQUS was only $3620.909 \mathrm{kN}$, and corresponded to a critical load factor of $749.091 \mathrm{kN}$. This difference of only $0.12 \%$ 363 is negligible and may have resulted from interpolations used by ABAQUS on the temperatures 364 located at nodes between adjacent segments, and/or truncation errors in the input form for the 365 applied loads. Figs. 7 through 10 illustrate the buckled shapes of the frame in Scenarios U, 2, 7 366 and 13, as obtained from the FEA model, respectively. Scenarios 2, 7 and 13 correspond to the 367 minimum time of failure resulting from blast damage applied to any segment on Columns 1, 2 368 and 3, respectively.
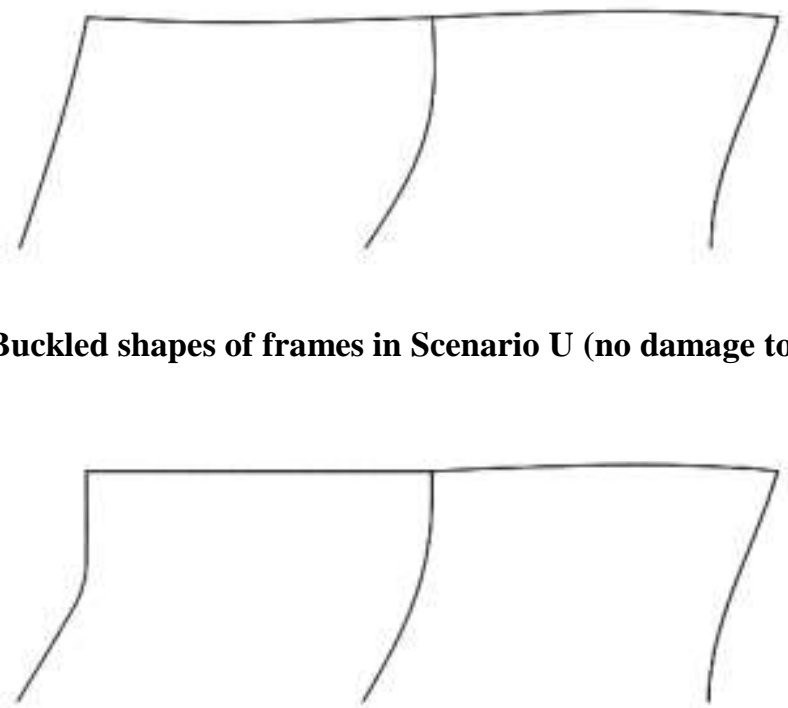
Figure 9 - Buckled shapes of frames in Scenario 7 (worst case delamination in Column 2)

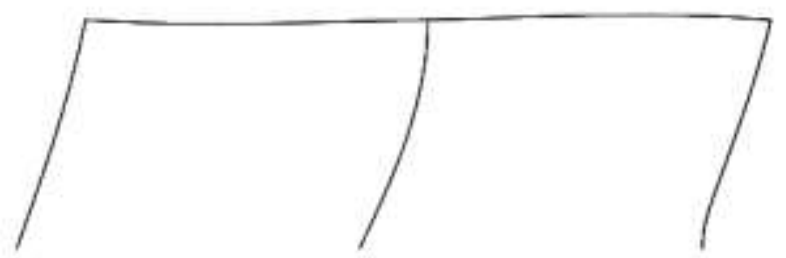

Figure 10 - Buckled shapes of frames in Scenario 13 (worst case delamination in Column 3)

From observing the buckled shapes in Figs. 7 to 10, it can be observed that a configuration similar to symmetric buckling exists in the beams. In fact, 27 of the 32 calibrated values of $v_{F N}$ obtained from the 16 scenarios (one for each beam) were negative. As such, $v_{F N}=-1$ may have been a more appropriate assumption in producing the un-calibrated results, although the resulting failure times only differ by $3.6 \%$ even with assuming $v_{F N}=1$. As such, the effect of $v_{F N}$ on the results of the failure time are not very significant in this example. Also, the column with the highest $P / P_{u}$ ratio in Table 2 appears to experience the most curvature in the buckled shape for each scenario, and the curvature becomes more severe as the corresponding $P / P_{u}$ ratio approaches unity. The implications of the $P / P_{u}$ ratio are further explained in the following paragraph.

In the un-calibrated analysis, the frame has a fire resistance of $138.8 \mathrm{~min}$ in the undamaged scenario. From Table 2, it can also be seen that damage to the insulation on the beams (Scenarios 4 through 6 and 10 through 12) has the least effect on the fire resistance of the frame. The fire resistance is affected to a greater extent if delamination of the fire protection occurs at the ends 
391 of the beams as opposed to in the middle, since the rotational rigidity of the beam-to-column

392 connections is reduced more quickly in these cases. Nevertheless, this reduction is not very

393 significant (up to $5.9 \%$ reduction for Scenario 12). Note that in the table, values of $P_{i} / P_{u, i}$ greater

394 than 0.9 indicate that individual column buckling is imminent, and that the lateral stiffness of the

395 frame is decreasing very quickly at the time of failure. However, it is noted that individual

396 column buckling cannot theoretically occur for non-lean-on columns as the lateral stiffness of the

397 individual column approaches negative infinity as $P$ approaches $P_{u}$. As such, the frame will

398 always buckle globally prior to the achievement of individual buckling load. Such is the case

399 when the insulation on any part of Column 2 is damaged (Scenarios 7 through 9), resulting in

400 failure as quickly as $101.4 \mathrm{~min}$. The damage to the insulation on the lower end of Column 2

401 (Scenario 7) is the worst scenario and represents a $26.9 \%$ decrease to the fire resistance of the

402 frame when compared with the undamaged case. It is also worth noting that damage to the

403 insulation near the fixed support (Scenario 13) also significantly reduces the fire resistance of the

404 frame to just $107.6 \mathrm{~min}$ (a $22.5 \%$ reduction). Overall, the results indicate that insulation damage

405 to Column 2 has the greatest reduction to the failure time of the frame, and it is clear that the

406 effect of blast damage to insulation can significantly reduce the fire resistance of a frame. From a

407 design standpoint, the results of the scenario analysis can be used to identify the most vulnerable

408 locations of a frame and increase the fire resistance in these locations by either strengthening the 409 members or providing more insulation.

\section{CONCLUSION}

411 Presented in this paper is a new method for computing the lateral stiffness of an unbraced semi-

412 rigid steel storey frame with three-segmented members, where the three segments in each

413 member can be set to have different input temperatures, or manually prescribed elastic modulus. 
414 The resulting lateral stiffness of the unbraced frame can then be computed. When the lateral

415 stiffness reaches zero, the frame becomes unstable. The proposed methodology can be applied

416 towards many modelling problems where non-linear or piece-wise temperature gradients occur

417 longitudinally in members. A numerical example is presented in which the effects of blast

418 damage to insulation during an ASTM E119 fire event are modelled via a member segment

419 containing delaminated insulation. The proposed method was also validated via finite element

420 analysis as it produces results that are virtually exact to the eigenvalue buckling analysis

421 approach when the value of $v_{N F}$ corresponding to the buckling mode is calibrated. Based on the

422 results of the numerical example, the location of the blast explosion can significantly influence

423 the fire resistance of a frame. The failure mode of the frame can also be changed between

424 individual column buckling depending on the location of fire or blast damage, which reinforces

425 the importance of considering different fire scenarios when analyzing structures.

\section{ACKNOWLEDGEMENTS}

427 The authors wish to thank the National Science and Engineering Research Council (NSERC)

428 [grant number RGPIN-203154-2013] of Canada for the financial support of this work.

\section{REFERENCES}

430 1. Toh WS, Fung TC, Tan KH. Fire resistance of steel frames using classical and numerical 431 methods. J Strc Engr 2000; 127(7):829-838.

432 2. Couto C, Real PV, Lopes N, Rodrigues P. Buckling analysis of braced and unbraced steel 433 frames exposed to fire. Engr Strc 2013; 49:541-559.

434 3. Xu L, Zhuang Y. Storey stability of unbraced steel frames subjected to non-uniform 435 elevated temperature distribution. Engr Strc 2014; 62-63:164-173. 
436 4. Rackauskaite E, Kotsovinos P, Rein G. Structural response of a steel-frame building to 437 horizontal and vertical travelling fires in multiple floors. Fire Safety J 2017; 91:542-552.

438 5. Dwaikat MMS, Kodur VKR. A simplified approach for predicting temperatures in fire 439 exposed steel members. Fire Safety J 2013; 55:87-96.

440 6. Xu L, Ma T, Zhuang Y. Storey-based stability of unbraced structural steel frames subjected 441 to variable fire loading. J Constr Steel Res 2018; 147:145-153.

442 7. Ziemian R. Guide to Stability Design Criteria for Metal Structures. 6th ed. Wiley \& Sons $443 \quad$ Ltd; 2010.

444 8. Yura J. The effective length of columns in unbraced frames. Engr J 1971; 8(2):37-42.

445 9. LeMessurier W. A practical method of second order analysis Part 2 - Rigid Frames. Engr J $446 \quad 1977 ; 14(2): 49-67$.

447 10. Lui EM. A novel approach for K factor determination. Engr J 1992; 29:150-159.

448 11. Aristizabal-Ochoa JD. Storey stability of braced, partially braced, and unbraced frames: $449 \quad$ classical approach. J Str Engr 1997; 123(6):799-807.

450 12. Xu L. The buckling loads of unbraced PR frames under non-proportional loading. J Constr $451 \quad$ Steel Res 2001; 58:443-465.

452 13. Tomecek DV, Milke JA. A study on the effect of partial loss of fire protection on the fire $453 \quad$ resistance of steel columns. Fire Tech 1993; 29(1): 3-21.

454 14. Arablouei A, Kodur VKR. Effect of fire insulation delamination on structural performance 455 of steel structures during fire following an earthquake or an explosion. Fire Safety J 2016; $456 \quad 84: 40-49$.

457 15. Braxtan NL, Pessiki SP. Postearthquake fire performance of sprayed fire-resistive material $458 \quad$ on steel moment frames. J Str Engr 2011: 137(9): 946-953. 
16. Wang WY, Li GQ, Kodur V. (2013). Approach for modeling fire insulation damage in steel columns. J Strc Engr, 139(4): 491-503.

461 17. Pettersson O, Magnusson S, Thor J. Fire engineering design of steel structures. Lund, 462 Sweden: Lund Institute of Technology; 1976.

463 18. Wickström U. Temperature calculation of insulated steel columns exposed to natural fire. $464 \quad$ Fire Safety J 1981-1982; 4(4):219-225.

465 19. BSI. BS EN 1993-1.2: 2005 Eurocode 3, Design of steel structures, Part 1.2: General rules - Structural fire design. London, UK: British Standards Institution; 2005.

20. Monforton G, Wu T. Matrix analysis of semi-rigidly connected frames. J Strc Div 1963; 89(6):13-42.

21. Chen KMG. Analysis of Semi-Rigid Connections Subjected to Fire Loads in a Steel Framework (MASc thesis), Waterloo, ON: University of Waterloo; 2010.

471 22. Al-Jabri KS, Burgess IW, Lennon T, Plank RJ. Moment-rotation-temperature curves for 472 semi-rigid joints. J Constr Steel Res 2005; 61: 281-303.

473 23. Ramberg W, Osgood WR. Description of stress-strain curves by 3 parameters. National 474 475 476 477 Advisory Committee for Aeronautics; 1943.

24. Xu L, Liu Y. Storey-based effective length factors for unbraced PR frames. Engr J 2002; 39(1):13-29.

25. Liu Y, Xu L. Storey-based stability analysis of multi-storey unbraced frames. Strc Engr Mech 2005; 19(6):679-705.

26. ASTM. Standard Test Methods for fire tests of building construction and materials (ASTM E119-16a). West Conshohocken, PA: American Society for Testing and Materials; 2016.

481 27. Lie TT. Structural Fire Protection. New York: American Society of Civil Engineers; 1992. 
482 28. Hoblit FM. Buckling load of a stepped column. J Aeronautical Sci 1951; 18(2):124-126.

483 29. Ypma TJ. Historical development of the Newton-Raphson method. SIAM Review 1995, $484 \quad 37(4): 531-551$.

485 30. Zhuang Y. Storey-based Stability Analysis of Unbraced Steel Frames at Ambient and 486 Elevated Temperatures (PhD thesis), Waterloo, ON: University of Waterloo; 2013. 


\section{Appendix A Thermal Restraints}

489 For fully restrained columns, the additional axial force $H_{i}$ may be calculated via Eqs. (A.1). Eqs.

490 (A.1) extend a similar derivation for two-segmented beams in $\mathrm{Xu}$ and Zhuang [3] to consider

491 three segments, and the tangent modulus theory from the Eurocode 3 [19] is also applied. In

492 utilizing Eq. (A.1) it is assumed that the differences in axial deformations among columns in the

493 same storey of the frame are ignored [3].

494

495

496

497

498

499

500

501

$$
\begin{gathered}
H_{i}=P_{i}-G_{i} \\
P_{i}=\frac{G_{i}+k_{i}\left(\Psi_{T}+\Psi_{M}\left(P_{i}\right)\right)}{1+\frac{k_{i} L_{c, i}}{A_{c, i} E_{0}}} \\
\Psi_{T}=L_{l, i} \int_{T_{0}}^{T_{l, i}} \alpha(T) d T+L_{m, i} \int_{T_{0}}^{T_{m, i}} \alpha(T) d T+L_{u, i} \int_{T_{0}}^{T_{u, i}} \alpha(T) d T \\
\Psi_{M}\left(P_{i}\right)=L_{l, i} \varepsilon_{M, l, i}\left(P_{i}\right)+L_{m, i} \varepsilon_{M, m, i}\left(P_{i}\right)+L_{u, i} \varepsilon_{M, u, i}\left(P_{i}\right)
\end{gathered}
$$

Where $A_{c, i}$ is the cross-sectional area of column $i$ and $\varepsilon_{M}\left(P_{i}\right)$ is the mechanical strain in the segment as obtained from the Eurocode 3 stress-strain curve in Eq. (A.2) [19]. The terms $\Psi_{T}$ and $\Psi_{M}$ refer to the thermal and mechanical deformations, respectively.

$$
\varepsilon_{M}(P)=\left\{\begin{array}{cl}
P / A_{c} E & ; P / A_{c} \leq f_{p, T} \\
\varepsilon_{y, T}-(a / b) \sqrt{b^{2}-\left(P / A_{c}+c-f_{p, T}\right)^{2}} & ; f_{p, T}<P / A_{c}<f_{t, Y} \\
\infty & ; P / A_{c} \geq f_{t, Y}
\end{array}\right.
$$

502 Where $a, b$, and $c$ are the parameters defined in Eurocode 3 [19], and $f_{p, T}$ and $f_{y, T}$ are the 503 proportional limit and yield stress tabulated in Eurocode 3 [19]. As the material may not always 504 be linearly elastic, solving Eq. (A.1a) requires an iteration procedure of computing $P_{i}$ and 505 converges readily if it is assumed that $P_{i}=G_{i}$ on the right-hand side of Eq. (A.1a) in the first iteration. In the numerical example, $H_{i}$ converges to within only $1.0 \mathrm{~N}$ within only four iterations. 
$\alpha$ is the coefficient of thermal expansion given in Eq. (A.3) [3] and $T_{0}$ is the ambient temperature and may be taken as $20^{\circ} \mathrm{C}$.

$$
\alpha(T)=(0.004 T+12) \times 10^{-6}{ }^{\circ} \mathrm{C}^{-1}
$$

510

511

512

513

$$
\begin{aligned}
& \eta_{0}=\tau_{N} \tau_{F}\left(1-r_{N}\right)\left(1-r_{F}\right) \mu_{N} \mu_{M} \mu_{F} \\
& \eta_{1}=3 r_{F}\left(1-r_{N}\right) \tau_{N}+3 r_{N}\left(1-r_{F}\right) \tau_{F} \\
& \eta_{2}=\eta_{1}+\frac{9 r_{N} r_{F}}{L_{b}}\left(\frac{L_{N}}{\mu_{N}}+\frac{L_{M}}{\mu_{M}}+\frac{L_{F}}{\mu_{F}}\right)
\end{aligned}
$$

$$
k_{i, j}=\frac{12 E_{0} I_{b}}{L_{b}{ }^{3}}\left(\frac{\eta_{2} \mu_{N} \mu_{M} \mu_{F}}{12 \eta_{0}-2 \lambda_{N F} \eta_{1}+6 \eta_{3}-9 r_{N} r_{F} \eta_{4}}\right)
$$

$$
\begin{aligned}
\eta_{3}=3 & r_{N} r_{F} \lambda_{0}+3 r_{F}\left(1-r_{N}\right) \tau_{N} \lambda_{F}+3 r_{N}\left(1-r_{F}\right) \tau_{F} \lambda_{N} \\
\eta_{4}=\frac{1}{L_{b}^{4}} & {\left[L_{N}^{3}\left(2 L_{b}-L_{N}\right) \frac{\mu_{M} \mu_{F}}{\mu_{N}}+L_{M}^{3}\left(2 L_{b}-L_{M}\right) \frac{\mu_{N} \mu_{F}}{\mu_{M}}+L_{F}^{3}\left(2 L_{b}-L_{F}\right) \frac{\mu_{N} \mu_{M}}{\mu_{F}}+\ldots\right.} \\
& 2\left(L_{N} L_{M}{ }^{3} \mu_{F}+L_{N} L_{F}{ }^{3} \mu_{M}+L_{M} L_{N}{ }^{3} \mu_{F}+L_{M} L_{F}{ }^{3} \mu_{N}+L_{F} L_{N}{ }^{3} \mu_{M}+L_{F} L_{M}{ }^{3} \mu_{N}\right)+\ldots \\
& 6\left(L_{N}{ }^{2} L_{M}{ }^{2} \mu_{F}+L_{N}{ }^{2} L_{F}{ }^{2} \mu_{M}+L_{M}{ }^{2} L_{F}{ }^{2} \mu_{N}\right)+\ldots \\
& 6\left(L_{N}{ }^{2} L_{M} L_{F}\left(\mu_{M}+\mu_{F}\right)+L_{M}{ }^{2} L_{N} L_{F}\left(\mu_{N}+\mu_{F}\right)+L_{F}{ }^{2} L_{N} L_{F}\left(\mu_{N}+\mu_{M}\right)\right]
\end{aligned}
$$




$$
\lambda_{N}=\frac{1}{L_{b}^{2}}\left[L_{N}^{2} \mu_{M} \mu_{F}+L_{M}^{2} \mu_{N} \mu_{F}+L_{F}^{2} \mu_{N} \mu_{M}+2\left(L_{N} L_{M} \mu_{M} \mu_{F}+L_{N} L_{F} \mu_{M} \mu_{F}+L_{M} L_{F} \mu_{N} \mu_{F}\right)\right]
$$

$$
\begin{aligned}
& \lambda_{F}=\frac{1}{L_{b}^{2}}\left[L_{N}^{2} \mu_{M} \mu_{F}+L_{M}^{2} \mu_{N} \mu_{F}+L_{F}^{2} \mu_{N} \mu_{M}+2\left(L_{N} L_{M} \mu_{N} \mu_{F}+L_{N} L_{F} \mu_{N} \mu_{M}+L_{M} L_{F} \mu_{N} \mu_{M}\right)\right] \\
& \lambda_{N F}=\frac{1}{L_{b}{ }^{3}}\left(L_{N}^{3} \mu_{M} \mu_{F}+L_{M}^{3} \mu_{N} \mu_{F}+L_{F}^{3} \mu_{N} \mu_{M}+\ldots\right.
\end{aligned}
$$

$$
\begin{aligned}
& 3 L_{N} L_{M}^{2} \mu_{N} \mu_{F}+3 L_{N} L_{F}^{2} \mu_{N} \mu_{M}+3 L_{M} L_{N}^{2} \mu_{M} \mu_{F}+\ldots \\
& 3 L_{F} L_{M}^{2} \mu_{N} \mu_{F}+3 L_{F} L_{N}^{2} \mu_{F} \mu_{M}+3 L_{M} L_{F}^{2} \mu_{N} \mu_{M}+\ldots \\
& \left.6 L_{N} L_{M} L_{F} \mu_{N} \mu_{F}\right)
\end{aligned}
$$

527 Note that since $H_{i}$ is a function of the elastic modulus, which can be a function of the axial load,

528 an iterative solution is required to determine the axial load of the column. However, since $H_{i}$ is

529 relatively small compared to $G_{i}$, the elastic modulus may be taken as a function of just the

530 gravity load rather than the total axial load without introducing significant errors. Doing this

531 prevents the need for an iterative solution, and simplifies the procedure of the time stability

532 assessment. 


\section{Appendix B Rotational Buckling}

The rotational buckling load of a column, $P_{b, i}$, can be determined by solving for the buckling condition implicitly using the approach outlined by Hoblit [28]. Consider the buckled shape of column $i$ in Fig. B.1. Once again, the subscript $i$ is removed from Fig. B.1 and subsequent equations referring to the variables and properties of this individual column.

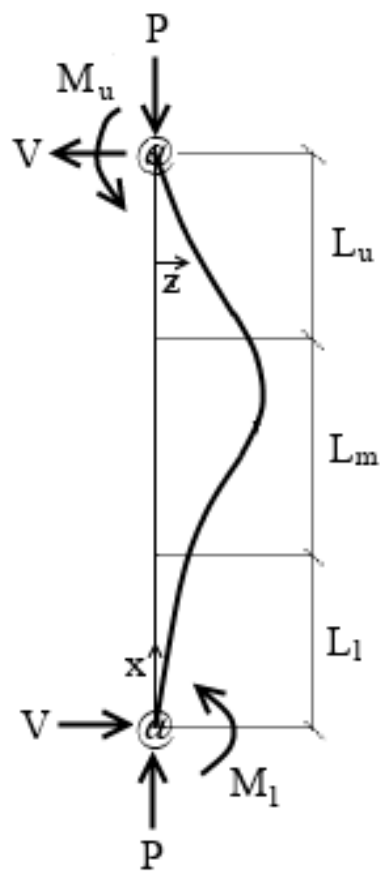

Figure B.1 - Buckled Geometry of a Three-Segment Column

The internal moment in each of the three sections of the column are given in Eqs. (B.1).

$$
\begin{gathered}
E_{l} I_{c} \frac{d^{2} z}{d x^{2}}=-P_{b} z(x)+M_{l}+V x ; \quad 0 \leq x \leq L_{l} \\
E_{m} I_{c} \frac{d^{2} z}{d x^{2}}=-P_{b}\left(z(x)-z\left(L_{l}\right)\right)+M\left(L_{l}\right)+V x ; \quad L_{l} \leq x \leq L_{l}+L_{m} \\
E_{u} I_{c} \frac{d^{2} z}{d x^{2}}=-P_{b}\left(z(x)-z\left(L_{l}+L_{m}\right)\right)+M\left(L_{l}+L_{m}\right)+V x ; \quad L_{l}+L_{m} \leq x \leq L_{c}
\end{gathered}
$$

By solving the differential equations in Eqs. (B.1), the bending moment, angle and deflection of the column can be obtained at the upper and lower ends of the column, as well as the points between adjacent segments. Thus, for buckling to occur, Eqs. (B.2) must be satisfied. 
Where the coefficients $\beta_{1}, \beta_{2}$ and $\beta_{3}$ are given in Eqs. (23).

$$
\begin{aligned}
\beta_{1}= & \phi_{l} \phi_{m} \phi_{u}\left(1-C_{l} C_{m} C_{u}\right)+\phi_{l}^{2} \phi_{u} S_{l} S_{m} C_{u}+\phi_{l}^{2} \phi_{m} S_{l} C_{m} S_{u}+\phi_{l} \phi_{m}^{2} C_{l} S_{m} S_{u} \\
& \beta_{2}=\phi_{l} \phi_{m} C_{l} C_{m} S_{u}+\phi_{l} \phi_{u} C_{l} S_{m} C_{u}+\phi_{m} \phi_{u} S_{l} C_{m} C_{u}-\phi_{m}^{2} S_{l} S_{m} S_{u} \\
\beta_{3}= & \phi_{l} \phi_{m} \phi_{u}+\phi_{m}^{2} S_{l} S_{m} S_{u}-\phi_{l} \phi_{m} C_{l} C_{m} S_{u}-\phi_{l} \phi_{u} C_{l} S_{m} C_{u}-\phi_{m} \phi_{u} S_{l} C_{m} C_{u}
\end{aligned}
$$

Where $\phi_{l}, \phi_{m}, \phi_{u}, S_{l}, S_{m}, S_{u}, C_{l}, C_{m}$ and $C_{u}$ are all shown in Eqs. (16). The lowest value of $P_{b}$ that satisfies Eqs. (B.2) may be taken as the final value $P_{b, i}$ for column $i$. The minimum value of $P_{b}$ satisfying the system of fourteen equations in Eqs. (B.2) can be obtained by using root-finding algorithms, such as the Newton-Raphson method [29]. 


\section{Appendix C End Fixity Factors for Three-Segment Members}

572 The end fixity factor for two-segmented members was previously derived by Zhuang [30]. A

573 new, similar derivation is presented in following for the end fixity factors of three-segment

574 members. Note that the derivation applies for both beams and columns. The end fixity factor is

575 defined as the ratio between the rotation at the end of the member, $\alpha$, and the combined rotation,

$576 \phi$, of the member and connection due to a unit end-moment, as shown in Fig. C.1.

577

578

579

580

581

582

583

584

585

586

587

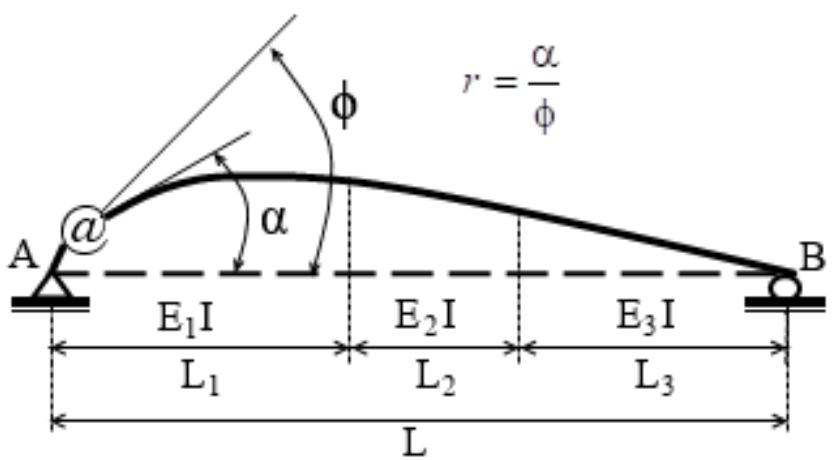

Figure C.1 - Definition of end fixity factor for a three-segmented member

Based on this relation, Zhuang [30] showed that the end fixity factor can be derived by determining the end rotation of an equivalent simply-supported member, $R_{S S}$, subjected to a unit end moment at the same end and substituting the result into Eq. (C.1).

$$
r=\frac{\alpha}{\phi}=\frac{R}{R+R_{S S}}
$$

Where $R$ is the rotational rigidity of the semi-rigid connection. For a member with uniform crosssection, $R_{S S}$ may be taken as $3 E I / L^{3}$, which results in the end fixity factors derived in [20].

Zhuang [30] showed that $R_{S S}$ for a two-segment member can be derived using the principle of virtual work. Using the same methodology, the principle of virtual work is henceforth applied towards three-segment members. Consider the simply supported member in Fig. C.2. 


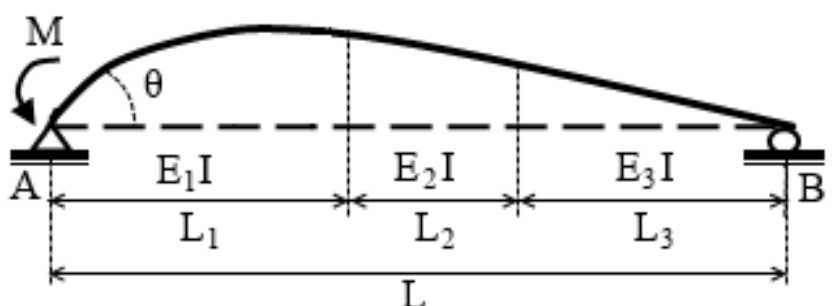

Figure C.2 - Equivalent simply-supported three-segment member subjected to unit end moment The virtual work principle is applied at the location of the end moment, $M$, on end $A$ of the member in Eq. (C.2).

$$
1 \times \theta=\int_{0}^{L_{1}} \frac{M x^{2}}{L^{2} E_{1} I} d x+\int_{L_{1}}^{L_{1}+L_{2}} \frac{M x^{2}}{L^{2} E_{2} I} d x+\int_{L_{1}+L_{2}}^{L} \frac{M x^{2}}{L^{2} E_{3} I} d x
$$

The value of the end rotation $\theta$ at $A$ is therefore obtained via integration in Eq. (C.2). The value of $R_{S S}$ can then be obtained by dividing the moment $M$ by $\theta$ and substituting the elastic modulus degradation factors from Eq. (1) to obtain Eq. (C.3).

$$
R_{S S}=\frac{M}{\theta}=\frac{3 E_{0} I}{L}\left(\frac{1}{\tau}\right)
$$

Where $\tau$ is an adjustment factor that accounts for the non-uniformity of the elastic modulus in the member, and is expressed in Eqs. (4) based on end moments being applied on the corresponding ends of the members. Thus, substituting Eq. (C.3) into Eq. (C.1) yields the end fixity factor equation in Eqs. (3). 


\section{Appendix D Equivalent Rotational Stiffness of Connecting Beams}

603 The rotational rigidity of a beam being connected to the end of a column is derived in this

604 appendix by utilizing the slope-deflection and conjugate-beam methods, similar to the approach

605 demonstrated in [20] but extended for three-segmented members. Consider first the deformation

606 of the beam shown in Fig. D.1.

607

608

609

610

611

612

613

614

615

\section{6} 617 Eqs. (D.2).

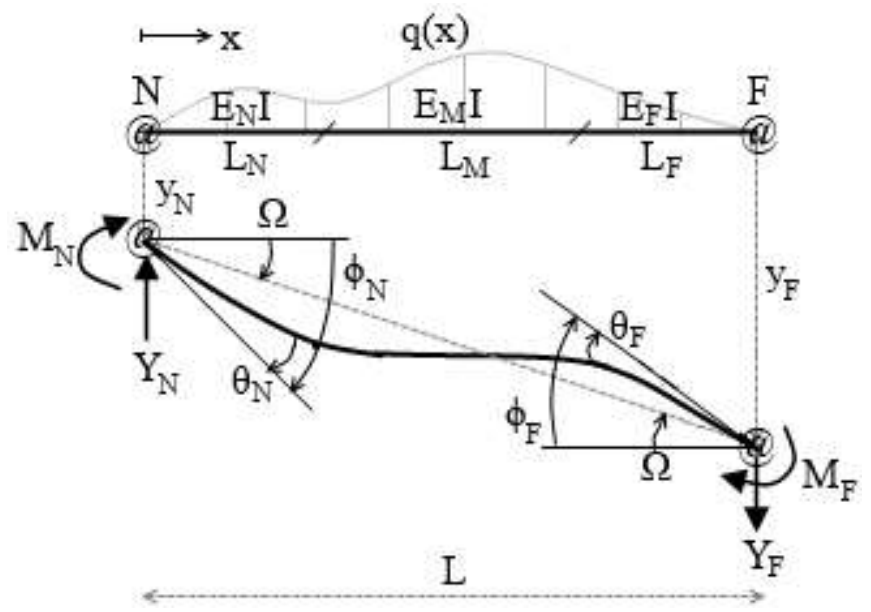

Figure D.1 - Generalized three-segment beam subjected to end moments

The near and far ends of the beam in Fig. D.1 are denoted as $N$ and $F$, respectively. The subscript $M$ denotes the middle segment of the beam. The displacement symbols $y, \phi, \theta$ and $\Omega$ correspond to the end deflection, rotation of the connection, net rotation between the member end and connection, and chord rotation, respectively. The force symbols $Y, q$ and $M$ correspond to the transverse reaction, transverse load function and end moments, respectively. Then the internal moment can be expressed in Eq. D.1.

$$
M(x)=\int_{0}^{x} \int_{0}^{x} q(x) d x^{2}+C_{1} x+C_{2}
$$

Where $C_{1}$ and $C_{2}$ are integration constants. The boundary conditions for Eq. (D.1) are given in 
620 Substituting the boundary conditions into Eq. (D.1) to solve for the constants results in the internal moment equation in Eq. (D.3).

$$
M(x)=\int_{0}^{x} \int_{0}^{x} q(x) d x^{2}+M_{N}\left(1-\frac{x}{L}\right)+M_{F}\left(\frac{x}{L}\right)-\frac{x}{L} \int_{0}^{L} \int_{0}^{L} q(x) d x^{2}
$$

Traditionally in stability analysis, loads are assumed to be directly applied to the columns and the only effect of the connected beam being considered is the rotational restraint [12]. As such, it is assumed that no transverse loads are applied on the beam between the ends $(q(x)=0)$. Then let $c(x)$ be obtained by dividing $M(x)$ by $E(x) I$, resulting in Eq. (D.4).

$$
c(x)=\frac{M_{N}}{E(x) I}\left(1-\frac{x}{L}\right)+\frac{M_{F}}{E(x) I}\left(\frac{x}{L}\right)
$$

Due to the piece-wise nature of $E(x)$ in the three-segment beam, $c(x)$ is piece-wise and can be split into individual functions of the local coordinates in each segment. Let the local coordinates

630

631

632

634 635 636 637 $x_{N}, x_{M}$ and $x_{F}$ correspond to the near, middle and far segments, given in Eqs. (D.5).

$$
\begin{gathered}
x_{N}=x ; \quad 0 \leq x_{N} \leq L_{N} \\
x_{M}=x-L_{N} ; \quad 0 \leq x_{M} \leq L_{M} \\
x_{F}=x-\left(L_{N}+L_{M}\right) ; \quad 0 \leq x_{F} \leq L_{F}
\end{gathered}
$$

Then the corresponding local functions $c$ are given in Eqs. (D.6).

$$
\begin{gathered}
c_{N}\left(x_{N}\right)=\frac{M_{N}}{\mu_{N} E_{0} I}\left(1-\frac{x_{N}}{L}\right)-\frac{M_{F}}{\mu_{N} E_{0} I}\left(\frac{x_{N}}{L}\right) \\
c_{M}\left(x_{M}\right)=\frac{M_{N}}{\mu_{M} E_{0} I}\left(1-\frac{L_{N}}{L}-\frac{x_{M}}{L}\right)-\frac{M_{F}}{\mu_{M} E_{0} I}\left(\frac{L_{N}}{L}+\frac{x_{M}}{L}\right) \\
c_{F}\left(x_{F}\right)=\frac{M_{N}}{\mu_{F} E_{0} I}\left(1-\frac{L_{N}}{L}-\frac{L_{M}}{L}-\frac{x_{F}}{L}\right)-\frac{M_{F}}{\mu_{F} E_{0} I}\left(\frac{L_{N}}{L}+\frac{L_{M}}{L}+\frac{x_{F}}{L}\right)
\end{gathered}
$$

Let $A_{N}, A_{M}$ and $A_{F}$ be the areas under the curves $c_{N}, c_{M}$ and $c_{F}$ over their corresponding domains, respectively, expressed in Eq. (D.7).

$$
A_{N}=\int_{0}^{L_{N}} c_{N}\left(x_{N}\right) d x_{N}
$$




$$
\begin{aligned}
A_{M} & =\int_{0}^{L_{M}} c_{M}\left(x_{M}\right) d x_{M} \\
A_{N} & =\int_{0}^{L_{N}} c_{N}\left(x_{N}\right) d x_{N}
\end{aligned}
$$

643 The total area under $c(x)$ is therefore $A=A_{N}+A_{M}+A_{F}$, and the centroid, $\bar{x}$, of $c(x)$ can be

644 expressed in Eq. (D.8).

645

646

648

649

650

651

652

653

654

655

656

657

658

659

660

$$
\bar{x}=\frac{\bar{x}_{N} A_{N}+\bar{x}_{M} A_{M}+\bar{x}_{F} A_{F}}{A}
$$

Where $\bar{x}_{N}, \bar{x}_{M}$ and $\bar{x}_{F}$ are the global $x$ coordinates of the centroids in each of the functions $c_{N}, c_{M}$ and $c_{F}$, respectively, and given in Eqs. (D.9).

$$
\begin{gathered}
\bar{x}_{N} A_{N}=\int_{0}^{L_{N}} x_{N} c_{N}\left(x_{N}\right) d x_{N} \\
\bar{x}_{M} A_{M}=\int_{0}^{L_{M}}\left(x_{M}+L_{N}\right) c_{M}\left(x_{M}\right) d x_{M} \\
\bar{x}_{F} A_{F}=\int_{0}^{L_{F}}\left(x_{F}+L_{N}+L_{M}\right) c_{F}\left(x_{F}\right) d x_{F}
\end{gathered}
$$

By the conjugate beam method, the rotations of the beam ends are related to $c$ by Eqs. (D.10).

$$
\begin{gathered}
\theta_{N}-\phi_{N}-\Omega=A\left(1-\frac{\bar{x}}{L}\right) \\
\theta_{F}-\phi_{F}-\Omega=-A\left(\frac{\bar{x}}{L}\right)
\end{gathered}
$$

Assume that $\Omega=0$, since the columns in the frame are not expected to experience significant differential axial deformations. Also, since the ends of the beam are semi-rigidly connected, $\phi$ can be expressed in terms of the end moments and rotational rigidities according to Eq. (D.11).

$$
\begin{aligned}
& \phi_{N}=M_{N} / R_{N} \\
& \phi_{F}=M_{F} / R_{F}
\end{aligned}
$$

Where $R$ can be obtained by rearranging Eqs. (3). Thus, substituting Eqs. (D.11) into Eqs. (D.10) and solving explicitly for $M_{N}$ yields the end moments given in Eqs. (D.12). 


$$
M_{N}=\frac{6 E_{0} I r_{N}}{L}\left[\frac{2 \tau_{F} \mu_{L} \mu_{M} \mu_{R}\left(1-r_{F}\right) \theta_{N}+2 \lambda_{N N} r_{F} \theta_{N}+\lambda_{N F} r_{F} \theta_{F}}{4 \lambda_{A}+r_{N} \lambda_{B}+r_{F} \lambda_{C}-r_{N} r_{F} \lambda_{D}}\right]
$$

662 Note that swapping the coefficients $N$ and $F$ in the terms of Eq. (12) yields $M_{F}$. Finally, the beam 663 rotational stiffness contribution to the end of the connected column, $R_{i, j}$ in Eq. (6), is obtained by 664 dividing Eq. (D.12) by $\theta_{N}$. 